Oral

\title{
Evaluation of High Resolution Melting Technique for Detection of JAK2-V617F Mutation in Formalin-Fixed Paraffin-Embedded Specimen from Myeloproliferative Neoplasm Cases
}

Mohammad Reza Abdullahi Abdul Saboor ${ }^{1}$, Nor Zamzila Abdullah ${ }^{1}$, Rosmawati Ismail $^{2}$, Naznin Muhammad ${ }^{1}$, Norlelawati A. Talib ${ }^{1}$

${ }^{1}$ Department of Pathology and Laboratory Medicine, Kulliyyah of Medicine, International Islamic University Malaysia

${ }^{2}$ Hospital Tengku Ampuan Afzan, Kuantan.

Presenter: Mohammad Reza Abdullahi Abdul Saboor

Introduction: Myeloproliferative neoplasm (MPN) is a group of myeloid disorder which lead to erythrocytosis, thrombocytosis and leukocytosis. MPN with BCR-ABL positive is chronic myeloid leukemia (CML) while BCR-ABL negative MPN includes polycythemia vera (PV), essential thrombocytemia (ET) and primary myelofibrosis (PMF). One of the major criteria for diagnosis of BCR-ABL negative MPN is the presence of JAK2-V617F mutation which is positive in $95 \%$ of PV and around $60 \%$ of ET and MF. Beside peripheral blood specimen, Formalin-Fixed Paraffin-Embedded (FFPE) marrow specimen can be used for detection of this mutation. Unfortunately, FFPE produces low quality DNA that put a challenge for successful amplification of DNA. We aimed to evaluate the utility of High Resolution Melting (HRM) analysis for detection of JAK2-V617F mutation in FFPE specimen from MPN cases. Materials and Methods: This study is a descriptive cross-sectional study. Forty FFPE marrow specimens were retrieved from the year 2014-2016. Bio-Rad Precision Melt Analysis software was used for analysis of HRM data. Allele-specific PCR was done for validation of results. Positive samples were subjected to Sanger sequencing. Results: JAK2-V617F mutation was positive in 3 out of 4 in PV, 0/2 in ET, 4/4 in PMF, 4/10 in non-classified MPN and 1/20 in CML. Level of agreement between HRM and AS-PCR was 97.5\%. Conclusion: HRM is a rapid and powerful diagnostic assay, which is suitable for detection of JAK2-V617F mutation in FFPE marrow specimen. 